\title{
Research on the Construction of Bachu Art Information Resources Qiang Liu
}

\author{
Art Academy, China Three Gorges University, Yichang, China
}

Hubei province university humanities and social science research base: Bachu Art Development Research Center

640690697@qq.com

\section{Keywords: Bachu art; Information resources; Database}

\begin{abstract}
The construction of Bachu art information resources includes the analysis of the contents and requirements of Bachu art information resources, the analysis of the types of Bachu art information resources and technical means, and the construction of Bachu art database. The construction of Bachu art database has been elaborated in detail the process of Bachu art database construction, the system structure of Bachu art database system, and the function module of Bachu art resource database.
\end{abstract}

\section{Introduction}

As a subset of art, Bachu art is an important part of Chinese culture. As the interaction, coupling the artistic hybridity examples, as the upper reaches of the Yangtze River culture and Wuling national cultural corridor with typical culture, "Bachu art" is a worthy of academic research field. However, the Bachu art research at home and abroad is still in the initial stage, there are still gaps in many parts and fracture. In view of the construction of information resources of Bachu art, there are no systematic research in the domestic and foreign. In the domestic occupy most of the market share of the Internet search engine Baidu search keywords "Bachu art", there is no system of professional information resources. In the international Internet search engine "GOOGLE" on the search "Art Bachu", in the world has not found the system information resources. In China the most authoritative academic journals "China HowNet" keyword search "Bachu art information resources, construction of artistic information resources study in Bachu found that academic achievement. Research on the construction of Bachu art information resources is of great significance.

\section{An Analysis of the Content and Demand of Bachu Art Information Resources}

\section{The Analysis of the Content of Bachu Art Information Resources}

According to the current study of Bachu art, the existing Bachu art can be divided into five categories: literature, art, music, dance, architecture. Although the current study of Bachu art is not systematic, the remains of the Bachu art is relatively fragmented, but there are more typical representative of each art species, emitting a unique and bright art light. Therefore, the content of Bachu art information resources should include these five categories, but also to organize the fragmented art content system.

Because of the particularity of art, Bachu art information resources as a subset of the information resources, besides the general resources in common, also has the following four special properties: first, art information carrier of different types, different, paper, film, cloth, pottery, bone discs, and even digital works of art and artists in itself, is a record carrier of artistic information resources. Second, art language is an important symbol of the art information resources, such as painting, music, language, dance, language, etc. Third, the art of information resources and the carrier of the contact, which means both record engraved, writing, printing, video and other traditional methods, the process includes artists and performers, such as painting, calligraphy, music, singing, dancing etc. Fourth, the content of the art information resources is rich, the value is lasting, has the multi discipline, and has the close relation with other discipline research achievement. [1] therefore, in the construction process of the Bachu art information resources, we should absorb the research 
achievements and methods of sociology, psychology, computer science, system science, philology, archaeology, anthropology and other disciplines, to enrich the artistic information resources in Bachu.

\section{Bachu Art Information Resources Demand Analysis}

Combined with the research status of Bachu art and the characteristics of Bachu art, Bachu art information resources should meet the following requirements:
a. A complete range of information resources, a variety of forms.
b. Organized and orderly, the structure of information resources system is reasonable.
c. Easy to share and spread.
d. Information resource query convenient and quick.
e. Can be updated in a timely manner.
f. Easy to manage.

\section{Analysis of the Types and Technical Means of the Art Information Resources in Bachu}

\section{The Types of Information Resources}

Bachu art information resources can be divided into two categories: physical information resources and digital resources.

Physical resources are information resources that exist in the form of entities. Bachu art physical resources include paper documents, art objects, etc. Although the current digital resources are the mainstream of the construction of resources, but in kind always has the digital resources can not replace the value and significance. In the construction of Bachu art information resources, the physical resources are essential. [2]

Digital resources are information resources that exist in the form of digital resources. Bachu art digital resources include text, images, audio, video, etc. Digital resources have a lot of advantages, to facilitate the preservation, sharing, sharing, display, exchange and dissemination of Bachu art, which provides a great convenience for the study of Bachu art. Digital resource is the important content of the construction of Bachu art information resources.

\section{Analysis of Technical Means}

Bachu art digital information resources, including information technology, information technology, information processing technology, information retrieval technology and information release technology.

Information collection technology, including text processing technology, image technology, audio technology, video technology, three-dimensional collection technology, etc.. [3] these technologies are the basis of the digital art information in Bachu, which can be used according to the characteristics of the art of Bachu. Such as Bachu ancient art and other text information can be used to collect text processing technology. Bachu art design, decoration and other images can be used to collect image technology. Bachu Arts in the dance, music, singing and other types of audio and video technology can be used to collect. Building, bronze ware, porcelain and other types of art in Bachu can use 3D acquisition technology to get the virtual information.

Information processing technology including information indexing and classification technology, metadata technology, information storage technology etc. Information indexing and classification technology enables the automatic identification and classification of Bachu artistic information resources, to form a word set, and lay the foundation for retrieval. Metadata technology can describe and locate the Bachu art information resources, extract the important features, and further data mining. Information storage technology can make the Bachu art information resources network, mass storage, with the help of cloud computing technology, can build a variety of information storage. [4]

Information retrieval technology can carry out full text search, intelligent search, accurate retrieval of Bachu art information resources. Information publishing technology can automatically organize the Bachu art information resources, and according to user needs, interests and habits of the automatic release of information and push. 


\section{Bachu Art Database Construction}

\section{The Construction Process of Bachu Art Database}

Bachu art database according to the needs of users to collect information, to provide users with accurate content, the two processing, convenient and flexible thematic information. Its main construction process is as follows:

a. Establish the principle, determine the scope of the information collection of the art resources database.

b. To strictly comply with the information collected by the digital standard and standardized system to form a standard digital document.

c. The use of metadata automatic indexing and artificial indexing hand generated digital documents.

d. To provide users with a network or stand-alone mode, and provide a database retrieval system including full text retrieval.

e. The maintenance of data and the addition of new data.

\section{The Architecture of Bachu Art Database System}

The system structure is an important part of information system, it is related to the preservation and management of information systems, communication, etc. to use, has good stability, scalability, maintainability, friendly and forward-looking. Bachu art database system uses three layers of $\mathrm{C} / \mathrm{S}$ (client / server) structure.

\section{Function Module of Bachu Art Resource Database}

The user of Bachu art resource bank can be divided into two kinds of administrators and general users. Corresponding to two different types of users, should have different function module design.

\subsubsection{The administrator user module}

Administrator user module functions are: to increase the user and delete users, increase resources, increase the record, upload statistics, users on the line and download statistics, view the latest upload and other functions.

The administrator is to add a new user to the user table by adding the function of the record, including setting the user name and password, and other information. In addition, in the "user" and "user interface," function, the administrator can all users to browse through this function has been authorized by the information, the administrator can according to the needs of the "delete" user. Similarly, if you find a user's information is incorrect, you can also modify the user information on the page by the "modification".

Administrators can add to the system according to the needs of the system of Bachu art resources, which in the process of adding resources, to add the details of the resources to add a complete. The operation of adding resources includes not only a new record in the resource table that is related to the resource information, but also a list of the resources to be added to the server to store the resource.

Add record function and most increase resource function is similar, the difference is: the increase in resources is also increasing record in the resource table will save resources from other places and uploaded to the server, you can set the resources available to download; and increase the record function is to add records related to the data in the table is not to the server add files.

As an administrator, the responsibility is not only necessary for the management of the Bachu art library this system, he also has a very important role, it is through the view of each user to login, database of resources to download a role for the resource in resource management.

\subsubsection{The general user module}

General user module functions are: free search, upload resources, classification list, and other functions.

The main page to open the Bachu art resources, no matter what type of users, are available through the input and resources in the name of the search keyword search resources; here is the search keyword fuzzy search, and can be one to three, if multiple keyword search, then the search process of each keyword is the relationship in logic and. Search results are displayed on a particular search page. 
The user can own good resources are uploaded to the server for other users to share, and administrator of additional resources here upload process is very similar, but also the basic information resources to fill, upload the file to the server and in the resource table add a record. Resources that do not meet the requirements will be deleted by the administrator.

Generally speaking, resources are stored in a lot of resources, and the user may not know or understand all the resources in the library, so for users, in addition to the keyword query method to search your own resources, but also through the "classification list" function to find the information they need. The function of the "classification list" is to display the categories of resources in the resource pool in the form of tables. Each resource in the resource pool has two categories: 1 and 2, respectively, which indicates the classification of the resource in the art. Users according to the information they want to find the classification of information, you will be able to find the answer to their satisfaction.

\section{Conclusion}

Bachu art variety, specific art form and characteristic, the construction of information resources can be arranged by the Bachu art summary, and preservation of information, can protect the existing permanent Bachu art heritage to the greatest extent. Using information technology, people can easily access to Bachu art resources, copy, spread, can span the time and space constraints on the exchange of Bachu art. Bachu art can also get more display space in the digital media. At the same time, the construction of information resources provides a more convenient way for the inheritance of Bachu art. Hope that this research can play a certain role in promoting the research and development of Bachu art.

\section{Acknowledgements}

2015 annual key research base of Humanities and Social Sciences in Universities in Bachu province Hubei Art Development Research Center open fund project (project number: 2015KF20).

\section{Reference}

[1] Lewis B R, Snyder C A, Rainer R K. An Empirical Assessment of the Information Resource Management Construct [J]. Journal of Management Information Systems, 2015, 12(1):199-223.

[2] Bogstad J M. The Librarian's Legal Companion for Licensing Information Resources and Services [J]. Collection Building, 2014, 30(3):445-446.

[3] Cena A, Gagolewski M, Mesiar R. Problems and challenges of information resources producers' clustering [J]. Journal of Informetrics, 2015, 9(2):273-284.

[4] Fountain J S, Reith D M, Holt A. The utilization of poisons information resources in Australasia. [J]. International Journal of Medical Informatics, 2014, 83(2):106-112. 